\title{
CD63 inhibits the cell migration and invasion ability of tongue squamous cell carcinoma
}

\author{
WEN-HUI LIU ${ }^{1,2^{*}}, \mathrm{XIN} \mathrm{LI}^{3 *}, \mathrm{XIN-LING} \mathrm{ZHU}{ }^{4}$, MEI-LING HOU ${ }^{1}$ and WEI ZHAO ${ }^{1}$ \\ ${ }^{1}$ School of Basic Medical Sciences, Jinzhou Medical University, Jinzhou, Liaoning 121200; ${ }^{2}$ Department of Dentistry, \\ Affiliated Hospital of Shaoxing University, Shaoxing, Zhejiang 312000; ${ }^{3}$ Department of Prosthodontics, \\ Second Affiliated Hospital of Jinzhou Medical University, Jinzhou, Liaoning 121200; ${ }^{4}$ Department of Periodontology, \\ Beijing Stomatological Hospital Capital Medical University, Beijing 100050, P.R. China
}

Received June 5, 2017; Accepted February 19, 2018

DOI: $10.3892 / 01.2018 .8499$

\begin{abstract}
The present study evaluated the expression and potential role of CD63 in the migration and invasion of tongue squamous cell carcinoma (TSCC) cells. Immunohistochemistry (IHC) was used to investigate the association between the expression level of CD63 protein and the histological differentiation of samples from 40 patients with TSCC and four normal tongue tissue specimens. RNA interference (RNAi) and gene transfection technology were used to alter the expression of CD63 in TCA8113 cells. The stable silencing and overexpression of CD63 in the TCA8113 cell line was used to assess the impact of the CD63 expression level on the migratory and invasive abilities of TCA8113 cells in a wound healing assay and a Transwell invasion assay. The effect of CD63 on the expression of matrix metalloproteinase (MMP)-2 and -9 were evaluated by western blot analysis. The results of IHC revealed a positive association between the CD63 expression level and the histopathological differentiation of TSCC and a negative association between the CD63 expression level and lymph node metastasis in TSCC. Western blotting revealed that the expressions of MMP-2 and MMP-9 were clearly upregulated in CD63-silenced TCA8113 cells but reduced in CD63-overexpressing TCA8113 cells, compared with the control. The wound-healing speed and the number of cells invading Matrigel-coated filters were negatively associated with the CD63 expression level. In summary, the results of the present study revealed that CD63 may be an inhibitor of TSCC malignancy and lymph node metastasis and may have applications in the prediction of prognosis and gene therapy for patients of TSCC.
\end{abstract}

Correspondence to: Professor Wei Zhao, School of Basic Medical Sciences, Jinzhou Medical University, 40 Songpo Road, Jinzhou, Liaoning 121200, P.R. China

E-mail: zhaowei8072@163.com

*Contributed equally

Key words: cluster of differentiation 63, tongue cancer, RNA interference, invasion, migration

\section{Introduction}

Oral squamous cell carcinoma (OSCC) was the tenth most frequently occurring solid cancer worldwide in 2015, and tongue squamous cell carcinoma (TSCC) is one of the leading causes of cancer-associated mortality in patients with oral cancer globally $(1,2)$. Despite the advances made in treatments for tongue cancer, including radical surgery and chemoradiotherapy, the 5-year survival rate of patients with TSCC remains poor, mainly due to the tendency of this cancer to affect lymph nodes and develop distant metastasis $(3,4)$. Although several studies regarding molecular mechanisms of TSCC progression have been performed, little is known regarding the molecular mechanisms underlying the regulation of metastatic dissemination (5-7).

The cluster of differentiation 63 (CD63) gene is located on human chromosome 12q13 and was the first tetraspan into be characterized (8). It is a member of the transmembrane 4 superfamily (TM4SF), which comprises heterogeneous membrane-bound glycoproteins that are expressed in endosomes and lysosomes and on the cell surface $(9,10)$. CD63 was first identified to be a protein strongly expressed on the cell surface in early-stage human melanoma cells, and was originally known as melanoma antigen 491 (ME491); it was observed within the complex network of internal membranes that are characteristic of late endosomes in mammalian cells $(8,11)$.

The trafficking of CD63 between the endosomal system and the cell surface is mediated by the clathrin adaptor protein 2 (AP2) complexes and caveolae or clathrin-coated pit-mediated endocytosis, a process that requires specific amino acid motifs present in the CD63 protein (10). Expression of transmembrane 4 L6 family member 5 (TM4SF5) is negatively correlated with that of CD63 in mouse fibrotic and human hepatic carcinoma tissues (12). CD63 is also known to be involved in the regulation of diverse cellular processes, including proliferation, adhesion, motility and differentiation (13). As malignant melanomas progress, expression of CD63 declines and the cells become more invasive $(8,14)$. Indeed, when the expression of CD63 in melanoma cells was silenced, their cell motility and matrix-degrading ability increased (15); however, when the recombinant vector pREP9-CD63 was transected 
in a CD63-negative melanoma cell line, the cell motility and metastatic capacity were reduced and the cells became more adhesive to the extracellular matrix (16).

CD63 was shown to serve a key role in the malignancy of cancer types, including lung adenocarcinoma, breast cancer, and colon cancer (17-19). Although CD63 is closely associated with multiple types of cancer development and progression, the functions of CD63 in TSCC have not been elucidated. Therefore, the present study investigated the roles of CD63 in the progression and development of TSCC using molecular and cell biology methods including immunohistochemistry, RNA interference (RNAi), gene transfection technology, wound healing and transwell invasion assays and western blotting.

\section{Materials and methods}

Cell culture. The human tongue squamous cell carcinoma TCA8113 cell line and 293 cells (Shanghai Institute for Cellular Biology, Chinese Academy of Sciences, Shanghai, China) were cultured in RPMI 1640 (Hyclone; GE Healthcare Life Science, Logan, UT, USA) and High Glucose DMEM Pyruvate medium (Hyclone; GE Healthcare Life Science), respectively, supplemented with $10 \%$ fetal bovine serum (FBS; Gibco; Thermo Fisher Scientific, Inc., Waltham, MA, USA), $100 \mathrm{U} / \mathrm{ml}$ penicillin, and $100 \mu \mathrm{g} / \mathrm{ml}$ streptomycin (Gibco; Thermo Fisher Scientific, Inc.) in $5 \% \mathrm{CO}_{2}$ at $37^{\circ} \mathrm{C}$.

Patients and tissue specimens. A total of 40 human TSCC tissues and four normal tongue tissue samples were examined. Among the 40 cases, there were 23 males and 17 females, with an age range from 36-86 years (median age, 55 years). The patients were histopathologically and clinically diagnosed at the Oral and Maxillofacial Surgery of the First Affiliated Hospital of Jinzhou Medical University (Jinzhou, China) between January of 2001 and April of 2017, and the pathological diagnosis was verified for each case. All patients had not received chemotherapy and radiotherapy prior to surgery. The present study was approved by the Ethics Committees of Jinzhou Medical University (approval nos. 20140005 and 20171105). Written informed consent was obtained from all patients stating their agreement to be involved in the study. All TSCC samples were staged according to the 2002 Union for International Cancer Control guidelines (20). Of the TSCC specimens, 16 cases were well-differentiated, 17 were moderately differentiated and 7 were poorly differentiated. A total of $11(27.5 \%)$ TSCC cases exhibited lymph node metastasis. The four normal tissue samples were collected from patients with tongue trauma. The collection of samples was performed in accordance with the policies of the National Research Ethics Committee, and informed consent was obtained from each patient. The clinicopathological features of the patients are summarized in Table I.

Immunohistochemistry. Immunohistochemical (IHC) analysis was performed to investigate the expression of CD63 in different grades of human TSCC samples using the Diaminobenzidene (DAB) Detection kit (Streptavidin-Biotin; cat. no. SP-9000-D; OriGene Technologies, Inc., Beijing, China) according to the manufacturer's instructions. The tissues were fixed in $10 \%$ formalin for $60 \mathrm{~min}$ at room temperature, paraffin-embedded, then deparaffinized in xylene for $10 \mathrm{~min}$. The xylene was replaced twice, each time tissue sections were soaked for $10 \mathrm{~min}$ at room temperature. The tissues were transferred to anhydrous ethanol for $5 \mathrm{~min}$, then $95 \%$ ethanol for $5 \mathrm{~min}$, and $75 \%$ ethanol for $5 \mathrm{~min}$ at room temperature. Then the tissues were rehydrated in water and rinsed in phosphate-buffered saline (PBS; pH 7.4) for $5 \mathrm{~min}$. Antigen retrieval was performed in a pressure cooker in citrate buffer (0.01 M, pH 6.0) for $15 \mathrm{~min}$, followed by treatment with $3 \%$ hydrogen peroxide for $15 \mathrm{~min}$ at room temperature and three washes with PBS for 2 min each time.

The specimens were incubated with antibody against CD63 (cat. no. ab216130; polyclonal rabbit; 1:50 dilution; Abcam, Cambridge, UK) for $1 \mathrm{~h}$ at $60^{\circ} \mathrm{C}$ and then washed three times with PBS (0.01 M). The specimens were then incubated with $100 \mu \mathrm{l}$ streptavidin-biotin-conjugated IgG antibody (cat. no. PV-6001, goat anti-rabbit; ready-to-use dilution; ZSGB-BIO; OriGene Technologies, Inc.) for $20 \mathrm{~min}$ at room temperature and washed three times with PBS. Streptavidin peroxidase was then applied to the specimens, and they were incubated for $10 \mathrm{~min}$ at room temperature. The incubation was followed by four rinses in PBS. Next, $30 \mu 1$ of DAB Chromogen was added to $1.5 \mathrm{ml}$ of DAB substrate, which were mixed by swirling and applied to the specimens, which were then incubated for $10 \mathrm{~min}$. The specimens were then washed four times with PBS.

The sections underwent counterstaining by Modified Harris Hematoxylin (0.5\% hematoxylin; cat. no. 6765003; Thermo Fisher Scientific, Inc.) for $5 \mathrm{~min}$ at room temperature according to the manufacturer's instructions, followed by dehydration in graded ethanol and mounting onto coverslips. The specimens were analyzed using a DFC310-FX light microscope (Leica Microsystems GmbH, Wechsler, Germany). Generally, each specimen was assigned a score according to the intensity of the staining $(0$, no staining; 1 , weak staining; 2 , medium staining; and 3 , strong staining), and the percentage of stained cells $(1,<10 \% ; 2,11-25 \% ; 3,26-50 \% ; 4,>50 \%)$. The final immunoreactive score was calculated as the mean of these two scores. When evaluating the protein expression of CD63, a score of $<2.5$ was defined as low and $\geq 2.5$ as high.

Screening of effective short hairpin RNAs (shRNAs) against CD63. For CD63 knockdown, three shRNA plasmids were synthesized to target the sequence of CD63 mRNA (NM_001780.5) by Shanghai GeneChem Co., Ltd. (Shanghai, China) (Table II). The 293 cells were cultured in two 6-well plates and transfected with shRNA using Lipofectamine 2000 (Invitrogen; Thermo Fisher Scientific, Inc.) following the transfection procedure; $48 \mathrm{~h}$ later, one 6-well plate of cells was harvested and the total RNA was isolated with RNAiso Plus (cat. no. 9108; Takara Biotechnology Co., Ltd., Beijing, China) according to the manufacturer's instructions.

cDNA was synthesized with the PrimeScript RT reagent kit (Takara Biotechnology Co., Ltd.). Reverse transcription-quantitative polymerase chain reaction (RT-qPCR) using a Thermal Cycler Dice Real Time System II with SYBR Premix Ex Taq II (Takara Biotechnology Co., Ltd.). The specific primers used were as follows: Human CD63 forward, 5'-CCCAAGCTT GCCACCATGGCGGTGGAAGGAGGAATGAAATG-3' and reverse, 5'-CCGCTCGAGCATCACCTCGTAGCCACTTCT 
Table I. Association between the CD63 expression in the 40 tongue squamous cell carcinoma tissue specimens and clinical characteristics.

\begin{tabular}{|c|c|c|c|c|}
\hline \multirow[b]{2}{*}{ Characteristic } & \multirow[b]{2}{*}{ Patients, n (\%) } & \multicolumn{2}{|c|}{ CD63 expression } & \multirow[b]{2}{*}{ P-value } \\
\hline & & Low & High & \\
\hline Male & $23(57.5)$ & 15 & 8 & 0.973 \\
\hline Female & $17(42.5)$ & 11 & 6 & \\
\hline \multicolumn{5}{|l|}{ Age, years } \\
\hline$>55$ & $22(55)$ & 15 & 7 & 0.744 \\
\hline$\leq 55$ & $18(45)$ & 11 & 7 & \\
\hline \multicolumn{5}{|c|}{ Histological differentiation } \\
\hline Well & $16(40)$ & 6 & 10 & 0.014 \\
\hline Moderate & $17(42.5)$ & 14 & 3 & \\
\hline Poor & $7(17.5)$ & 6 & 1 & \\
\hline \multicolumn{5}{|l|}{ TNM stage } \\
\hline I-II & $24(60)$ & 12 & 12 & 0.02 \\
\hline III-IV & $16(40)$ & 14 & 2 & \\
\hline \multicolumn{5}{|c|}{ Lymph node metastasis } \\
\hline $\mathrm{N}^{+}$ & $11(27.5)$ & 11 & 0 & 0.007 \\
\hline $\mathrm{N}^{-}$ & $29(72.5)$ & 17 & 14 & \\
\hline
\end{tabular}

CD63, cluster of differentiation 63; TNM, Tumor-Node-Metastasis.

GATAC-3'; and human $\beta$-actin forward, 5'-GCATCCACG AAACTACATTCAACTC-3' and reverse, 5'- CACTGTGTT GGCATAGAGGTCTTTG-3'.

All reactions were performed in triplicate. The data are expressed as the mean normalized expression (MNE). The MNE is directly proportional to the amount of RNA of the target gene relative to the amount of RNA of the reference gene $\beta$-actin. Analysis of relative gene expression data using the $2^{-\Delta \Delta \mathrm{Ct}}$ method (21).

The other 6-well plate of cells was harvested $48 \mathrm{~h}$ after transfection, and the protein was extracted using radioimmunoprecipitation assay (RIPA) buffer and $1 \%$ phenylmethylsulfonyl fluoride (PMSF) (both Beyotime Institute of Biotechnology, Haimen, China). The bicinchoninic acid (BCA) method was used to measure the concentration of the protein with a BCA Protein Assay kit (Aidlab Biotechnologies Co., Ltd., Beijing, China). Equivalent amounts of protein $(50 \mu \mathrm{g})$ were denatured in SDS sample buffer (cat. no. S9788; Sigma-Aldrich; Merck KGaA, Darmstadt, Germany) and separated by $10 \%$ SDS-PAGE and then transferred to a polyvinylidene difluoride (PVDF) membrane (Merck KGaA, Darmstadt, Germany). The membrane was blocked with $1 \%$ bovine serum albumin (BSA) (Sigma-Aldrich; Merck KGaA) for $2 \mathrm{~h}$ and incubated with the following primary antibodies: rabbit polyclonal anti-CD63 (cat. no. ab216130; 1:1,000 dilution; Abcam), mouse polyclonal anti- $\beta$-actin (cat. no. 3700; 1:5,000 dilution; Cell Signaling Technology, Inc., Danvers, MA, USA) overnight at $4^{\circ} \mathrm{C}$, and the signal was detected using horseradish peroxidase (HRP)-conjugated secondary antibodies (goat anti-rabbit IgG-HRP, cat. no. sc-2004; goat anti-mouse IgG-HRP, cat. no. sc-2005, 1:2,000 dilution; Santa Cruz Biotechnology, Inc., Dallas, TX, USA) followed by development using a Alkaline phosphatase substrate chromogenic kit (cat. no. PP2501; Aidlab Biotechnologies Co., Ltd.). The images were captured and analyzed using Omega Lum ${ }^{\mathrm{TM}} \mathrm{G}$ capture software (version 2.0.1027.0; Gel Company, San Francisco, CA, USA).

Construction of the CD63 overexpression plasmid. Total RNA was extracted from TCA8113 cells using RNAiso Plus (cat. no. 9108; Takara Biotechnology Co., Ltd.) according to the manufacturer's instructions. cDNA was synthesized with the PrimeScript RT reagent kit and 100 ng cDNA was used as a template for amplifying the CD63 gene using PrimeSTAR ${ }^{\circledR}$ HS Premix (cat. no. R040A; Takara Biotechnology Co., Ltd.) according to the manufacturer's protocol (the primers used were those aforementioned). The 50- $\mu 1$ reaction system contained $25 \mu \mathrm{l}$ PrimeSTAR HS Premix, $1 \mu \mathrm{l}$ each of the forward and reverse primers of CD63, $2 \mu \mathrm{l}$ cDNA, and $21 \mu \mathrm{l}$ distilled water.

The PCR thermocycling conditions were as follows: $98^{\circ} \mathrm{C}$ for $10 \mathrm{sec}, 55^{\circ} \mathrm{C}$ for $5 \mathrm{sec}, 72^{\circ} \mathrm{C}$ for $1 \mathrm{~min}$ and 30 cycles later $72^{\circ} \mathrm{C}$ for $10 \mathrm{~min}$. The CD63 gene was detected by $1 \%$ agarose gel electrophoresis and purified with an AxyPrep DNA Gel Extraction kit (Axygen; Corning Incorporated, Corning, NY, USA) according to the manufacturer's protocol. The purified CD63 gene was then subcloned into the HindIII/XhoI sites of the PEGFP-N3 vector (BioVector NTCC, Inc., Beijing, China). The recombinant plasmids were transformed into Escherichia coli DH5 $\alpha$ and screened by kanamycin (100 $\mu \mathrm{g} / \mathrm{ml}$; Sangon Biotech Co., Ltd., Shanghai, China). An Axyprep-96 Plasmid kit (Axygen) to purify the recombinant plasmids from bacterial cells cultured in lysogeny broth medium overnight. The recombinant plasmids were detected by restriction enzyme digestion and then DNA sequencing (Sangon Biotech Co., Ltd.). 
Transfection and selection of stable clones. The most effective shRNA plasmid against CD63 (named CD63-RNAi-4041) and the CD63 overexpressing plasmid (named PEGFP-N3-CD63) were transfected into TCA8113 cells using Lipofectamine 2000 and screened using geneticin (Gibco; Thermo Fisher Scientific, Inc.) at $600 \mu \mathrm{g} / \mathrm{ml}$. Neomycin-resistant clones were obtained, and the CD63 expression level was detected by western blot. An IHC assay was performed as aforementioned to observe the expression and location of CD63 in screened cell lines. The screened TCA8113 cells and normal cells were seeded in glass-bottom cell culture dishes and fixed in $4 \%$ paraformaldehyde at room temperature (Sangon Biotech Co., Ltd.) for $30 \mathrm{~min}$.

Following three washes with PBS for 5 min each time, the cells were treated with $0.1 \%$ Triton X-100 (Sigma-Aldrich) for $10 \mathrm{~min}$ and then washed three times with PBS. The cells were blocked in $1 \%$ BSA for $1 \mathrm{~h}$ and incubated with the rabbit polyclonal anti-CD63 antibody (1:1,000 dilution; Abcam) for $1 \mathrm{~h}$ at $37^{\circ} \mathrm{C}$. After being washed with PBS three times, the cells were incubated with the fluorescein isothiocyanate-conjugated anti-R-Phycoerythrin antibody (1:500 dilution; cat. no. ab34723; Abcam) for $30 \mathrm{~min}$, followed by three washes with PBS. The expression and location of CD63 protein were observed using a fluorescence microscope (magnification, x400; FSX100; Olympus, Shanghai, China).

Wound-healing assay. The transfected cells and normal TCA8113 cells ( $5 \times 10^{5}$ cells per well) were seeded in 24 -well plates, and when the cells reached a confluent state the cell layer was scratched with a sterile $200-\mu 1$ pipette tip. The medium and cell debris was aspirated away and replaced with $1 \mathrm{ml}$ of fresh RPMI 1640 medium without FBS. Images of the wounded area were captured at 0 and $24 \mathrm{~h}$, using a DMI3000 B light microscope (Leica Microsystems GmbH). The wound healing speed was calculated as the difference in the area between 0 and $24 \mathrm{~h}$ divided by the height of the wound, with the use of ImageJ1.46r software (National Institutes of Health, Bethesda, MD, USA).

Transwell cell invasion assay. The Transwell invasion assay was performed to examine the invasion ability of CD63-silenced and CD63-overexpressing TCA8113 cells, using a $6.5-\mathrm{mm}$ Transwell with an $8.0-\mu \mathrm{m}$ Pore Polyester Membrane Insert (Corning Incorporated) coated with $10 \mu 1$ Matrigel $\left(50 \mu 1 / \mathrm{cm}^{2}\right.$; Corning Incorporated). A total of $1 \times 10^{5}$ cells were plated into the upper chamber of the Transwell with $500 \mu 1$ RPMI 1640 medium without FBS, and $500 \mu 1$ RPMI 1640 medium with $10 \%$ FBS was added into the lower chamber. The cells were cultured for $24 \mathrm{~h}$ in $5 \% \mathrm{CO}_{2}$ at $37^{\circ} \mathrm{C}$.

The non-invading cells in the upper side of the filter were then gently removed with a soft cotton swab, and the cells that had invaded to the lower side of the filter were fixed with $4 \%$ paraformaldehyde at room temperature for $30 \mathrm{~min}$ and stained with $1 \%$ crystal violet at $37^{\circ} \mathrm{C}$ for $15 \mathrm{~min}$ (Sigma-Aldrich; Merck $\mathrm{KGaA}$ ). The number of cells in three randomly selected fields was counted with an Image Analysis System (version 3.3.0; Leica Microsystems $\mathrm{GmbH}$ ), and these numbers are expressed as the average number of migrating cells.

Assessing expression of matrix metalloproteinase-2 (MMP-2) and MMP-9. The stably transfected cell lines were lysed with 
RIPA and 1\% PMSF, and western blot analysis was performed following BCA protein analysis, performed as aforementioned. Following $10 \%$ SDS-PAGE (50 $\mu \mathrm{g}$ protein per lane) electrophoresis and the transfer of the protein to a PVDF membrane, the membrane was blocked with $1 \%$ BSA for 2 hat room temperature and incubated with the following primary antibodies: Rabbit polyclonal anti-MMP-2 (cat. no. 40994; 1:1,000 dilution; Cell Signaling Technology, Inc.), rabbit polyclonal anti-MMP-9 (cat. no. 2270; 1:1,000 dilution; Cell Signaling Technology, Inc.), and mouse polyclonal anti- $\beta$-actin (cat. no. 3700; 1:1,000 dilution; Cell Signaling Technology, Inc.) overnight at $4^{\circ} \mathrm{C}$. The signal was then detected using HRP-conjugated secondary antibodies (goat anti-rabbit IgG-HRP; cat. no. sc-2004; goat anti-mouse IgG-HRP, cat. no. sc-2005; 1:2,000 dilution; both from Santa Cruz Biotechnology, Inc.) for 1 hat room temperature followed by visualization using an Alkaline phosphatase substrate chromogenic kit (cat. no. PP2501; Aidlab Biotechnologies Co., Ltd.). The images were captured and analyzed using an Omega Lum $\mathrm{G}$ capture software.

Statistical analysis. Quantitative data are expressed as the mean \pm standard error of the mean. Statistical comparisons were performed using one-way analysis of variance followed by the Student-Newman-Keuls test. The $\chi^{2}$ test was used to analyze the association between CD63 expression and clinicopathological patient characteristics of TSCC. All statistical analyses were performed using the SPSS Statistics 18.0 software program (SPSS, Inc., Chicago, IL, USA). $\mathrm{P}<0.05$ was considered to indicate a statistically significant difference.

\section{Results}

Expression of CD63 in TSCC tissues. The IHC staining revealed a significant decrease in CD63 protein levels in the TSCC tissues compared with the normal tongue tissues (Table III). As shown in Fig. 1, in the normal tongue tissues, staining for the CD63 protein was deep brown-yellow-colored, granular and expressed mainly in the cell membrane and cytoplasm. In the TSCC tissue (Fig. 1A), the staining for the CD63 protein was weaker than that in the normal tongue tissue as the differentiation degree decreased significantly $(\mathrm{P}<0.05)$, indicating a positive association between the CD63 expression level and the histopathological differentiation of the tongue cancer (Fig. 1B).

CD63 protein expression in the stage I-II TSCC tissues was significantly higher than that in the stage III-IV TSCC tissues $(\mathrm{P}<0.05)$; the expression was significantly higher in the well- and moderately differentiated TSCC tissues compared with that in the poorly differentiated TSCC tissues $(\mathrm{P}<0.05)$. Lower expression of CD63 was significantly associated with lymph node metastasis $(\mathrm{P}<0.01)$. Thus, the protein expression level of CD63 in TSCC was significantly associated with the Tumor-Node-Metastasis stage (20), tumor differentiation and lymph node metastasis, although it was not associated with the age or sex of the patient (Table I).

Screening of effective shRNA plasmids against CD63. The shRNA plasmids were efficiently transfected into 293 cells with the use of Lipofectamine 2000; RT-qPCR and western
Table III. Protein expression of CD63 in TSCC tissues $(n=40)$ and normal tongue tissues $(n=4)$.

\begin{tabular}{lrrrrr}
\hline & \multicolumn{4}{c}{ CD63 } \\
Histological type & $\mathrm{n}$ & Low & High & Ratio, \% & P-value \\
\cline { 3 - 5 } Normal tissues & 4 & 0 & 4 & 100 & 0.023 \\
TSCC tissues & 40 & 26 & 14 & 35 & \\
\hline
\end{tabular}

CD63, cluster of differentiation 63; TSCC, tongue squamous cell carcinoma.

blot analysis results revealed that all three shRNA plasmids effectively silenced the expression of CD63, with the plasmid CD63-RNAi-4041 being the most efficient. The interference efficiency of CD63-RNAi-4041 at the mRNA and protein levels was 88 and $74 \%$, respectively $(\mathrm{P}<0.05$; Fig. 2).

Construction of the CD63-overexpressing plasmid. The CD63 gene sequence was obtained by PCR, from which a specific 717-bp band was observed (Fig. 3A). PEGFP-N3-CD63 plasmids were successfully obtained following restriction enzyme digestion (Fig. 3B) and DNA sequencing.

Selection of stable clones. Following screening using geneticin, stable clones transfected with CD63-RNAi-4041 and PEGFP-N3-CD63 plasmids were obtained. Western blotting revealed that the expression level of CD63 in TCA8113 cells transfected with CD63-RNAi-4041 was 59\% lower than that in the controls, and the expression level of CD63 in cells transfected with PEGFP-N3-CD63 was 32\% higher than that in the controls; these differences were significant $(\mathrm{P}<0.05$; Fig. $4 \mathrm{~A}$ and B). The CD63-silenced TCA8113 cell line CD63-low (CD63-L) and the CD63 overexpressing cell line CD63-high (CD63-H).

The indirect immunofluorescence staining observed using confocal microscopy revealed that the CD63 protein was primarily located in the outer membrane and cytoplasm of TCA8113 cells. The fluorescent signal intensity in the CD63-H cells was the strongest of the three cell lines, and that in the CD63-L cells was the weakest. This difference in the fluorescence signal may be representative of the expression level of CD63 protein (Fig. 4C).

CD63 expression inhibits TCA8113 cell migration and invasion. The CD63-L cells were able to repair the wound $73 \%$ more rapidly than the control cells, whereas the speed of repair of CD63-H cells was $59 \%$ slower than that of the control cells $(\mathrm{P}<0.05$; Fig. $4 \mathrm{~A}$ and $\mathrm{B})$. The invasion assay revealed that $70 \%$ more $\mathrm{CD} 63-\mathrm{L}$ cells invaded through the Matrigel-coated filters compared to the control cells, whereas 71\% fewerCD63-H cells invaded compared to the control cells (Fig. 4C and D). There was a significant negative association between the CD63 expression level and the migratory and invasive ability of TCA8113 cells $(\mathrm{P}<0.05)$.

The results of western blotting revealed that the expressions of MMP-2 (Fig. 4E and F) and MMP-9 (Fig. 4G and H) were 

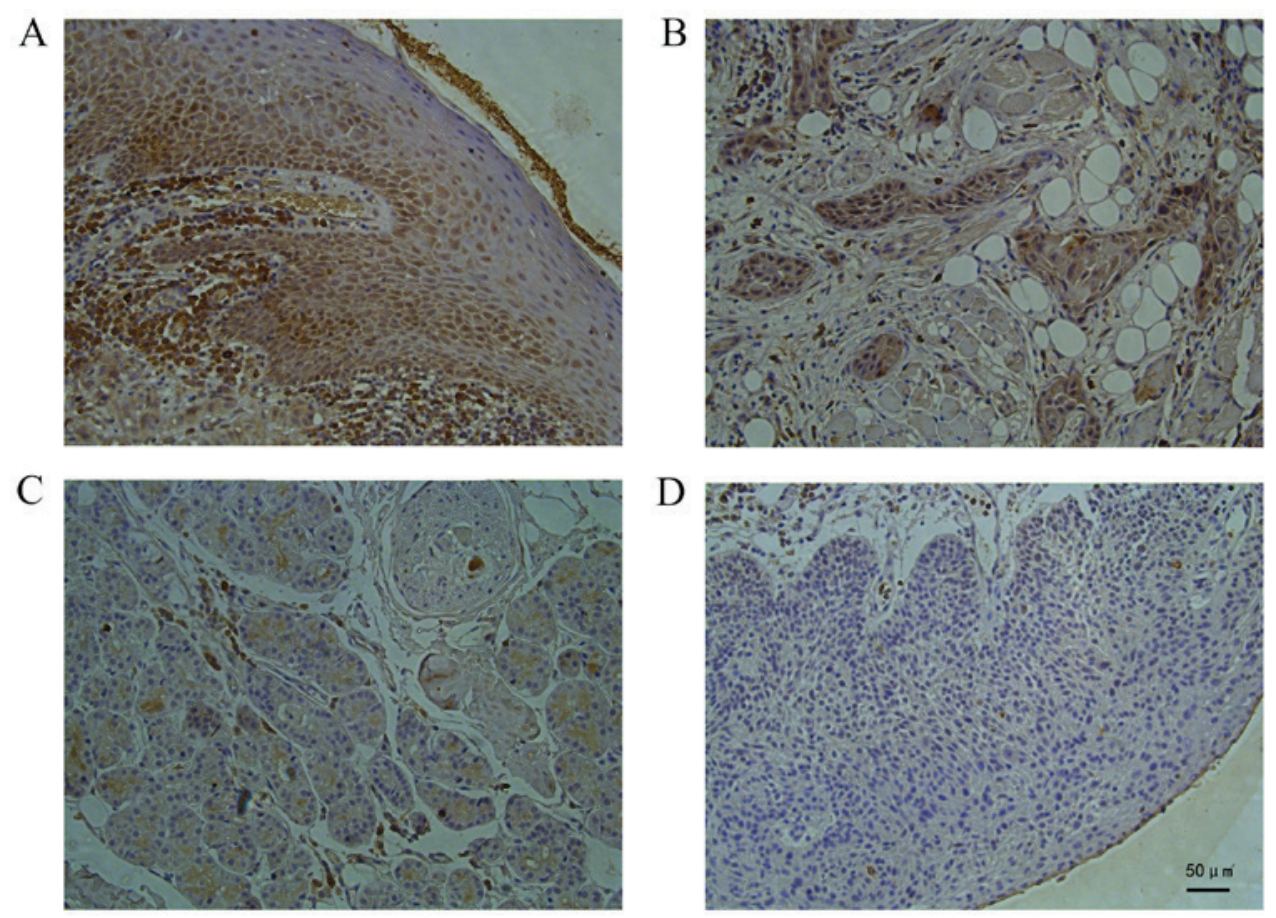

Figure 1. Immunohistochemistry result depicting the typical expression of CD63 in normal tongue tissues and TSCC. (A) Normal tongue tissue. (B) Well-differentiated TSCC. (C) Moderately differentiated TSCC. (D) Poorly differentiated TSCC. Scale bar, $50 \mu$ m. CD63, cluster of differentiation 63; TSCC, tongue squamous cell carcinoma.

upregulated by 60 and 61\%, respectively, in CD63-L cells, but were downregulated by 29 and 41\%, respectively, in CD63-H cells, compared with the control cells. Taken together, the findings of the present study indicated that CD63 might serve a role in inhibiting the migration and invasion of TSCC cells.

\section{Discussion}

TSCC is a threat to human health worldwide, and its metastasis is believed to be one of the fundamental features that contributes to the majority of incidences of cancer-associated mortality in humans $(1,2)$. However, the detailed molecular mechanisms of TSCC remain elusive. CD63 expression is associated with the biological behavior of solid tumors, particularly those with metastatic potential, and it has been found to serve an inhibitory role in the invasion and metastasis of multiple tumor types. Kwon et al (17) examined the expression level of CD63 in 90 cases of non-small cell lung cancer (NSCLC) to investigate the potential of CD63 as a prognostic biomarker for lung cancer subtypes, using tissue microarray-based immunohistochemistry. The results of this analysis revealed that $63.3 \%$ of the NSCLC samples were CD63-negative, and the CD63 protein level was lower than that in normal tissue. CD63 protein negativity was significantly associated with larger tumor size, advanced clinicopathological stage and poor patient survival rates $(\mathrm{P}=0.008)$; the findings of this study (17) indicated that CD63 could be used as a biomarker to predict the prognosis of patients with early-stages of lung adenocarcinoma.

Woegerbauer et al (22) used the immunohistochemistry staining of Merkel cell carcinoma specimens from 25 patients and observed that CD63 expression was significantly associated with the disease-free survival time of patients. In addition, a study by Chen et al (23) demonstrated that CD63
A

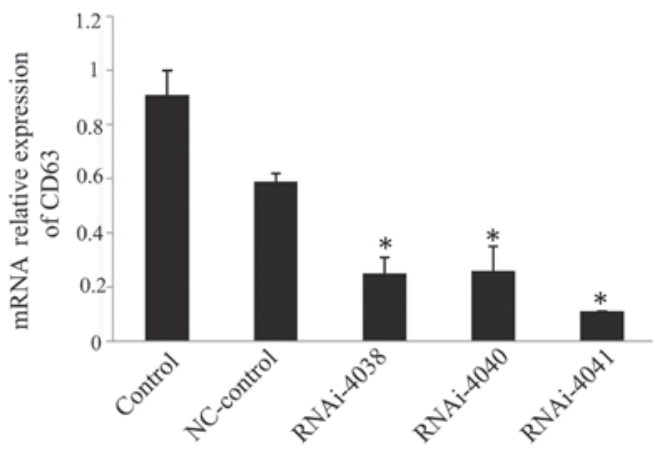

B

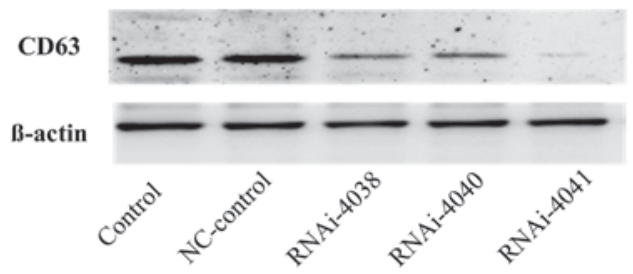

C

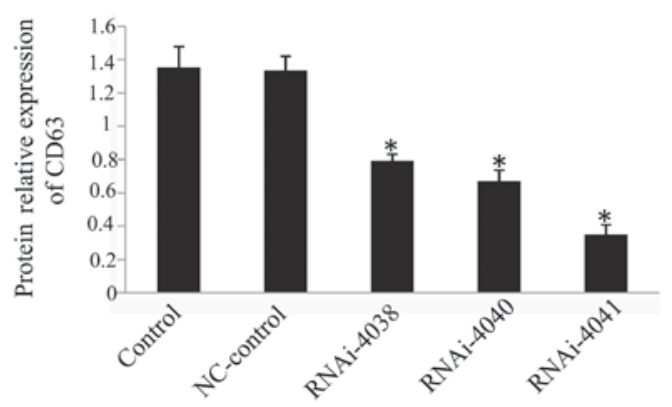

Figure 2. Screening of effective shRNA plasmids against CD63. (A) Reverse transcription-quantitative polymerase chain reaction analysis of CD63 expression in shRNA-transfected 293 cells. (B) Western blot analysis of CD63 expression in shRNA-transfected 293 cells, with (C) quantification. Data are the mean \pm standard deviation. ${ }^{~} \mathrm{P}<0.05$ vs. control cells. shRNA, short hairpin RNA; CD63, cluster of differentiation 63. 
A

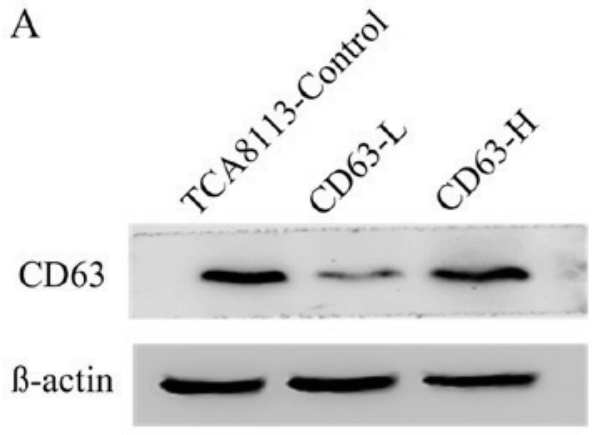

B

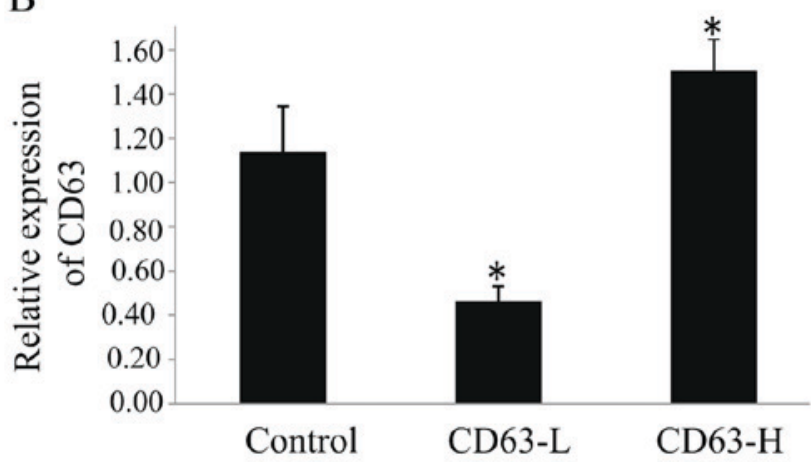

C Control

CD63-L CD63-H
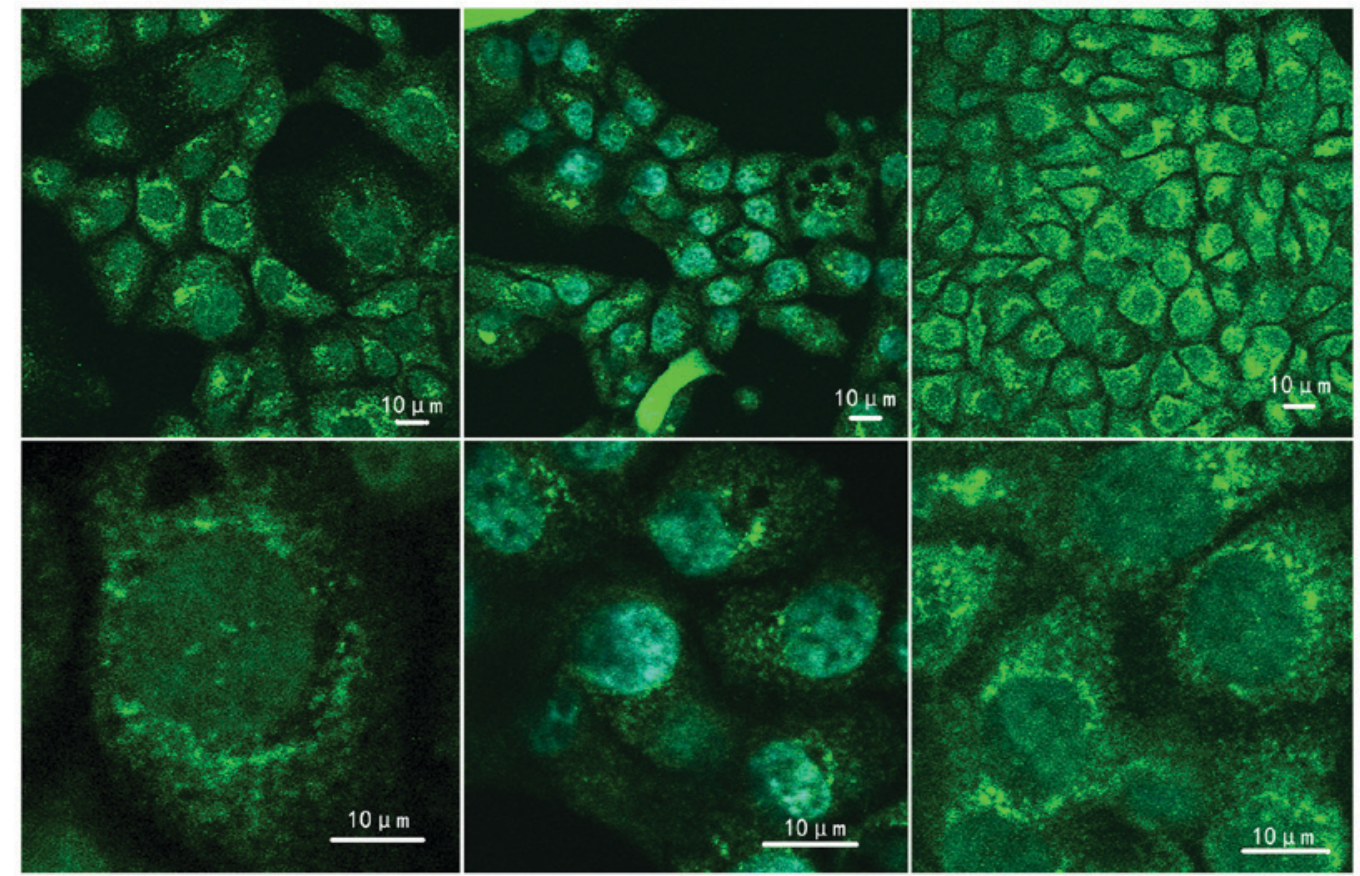

Figure 3. Expression and location of CD63 in selected cell lines. (A) The relative expression of CD63 in CD63-silenced and CD63-overexpressing TCA8113 cells, (B) quantification of the relative expression of CD63. "P<0.05 vs. control. (C) The expression and location of CD63 in the three TCA8113 cell lines shown by immunofluorescence staining. Scale bar, $10 \mu \mathrm{m}$. Control, TCA8113 cells untransfected exogenous CD63; CD63-L, low CD63 expression; CD63-H, high CD63 expression.

was strongly expressed in all normal gastric epithelium and gastric ulcer tissues, and M0-stage gastric carcinomas exhibited stronger expression of CD63 than M1-stage carcinomas. Chen et al (23) also noted that reductions in the expression of CD9, CD63 and CD82 were indicators of the metastatic potential of gastric carcinoma cells, and proposed that constitutive expression of CD63 may indicate that CD63serves a direct role in human gastric carcinogenesis (23).

Similar results to those discussed in the previous paragraph were achieved in the present study: Immunohistochemical staining revealed that CD63 expression was downregulated in TSSC tissues, and analysis of the clinicopathological characteristics of the TSCC patients indicated that CD63 expression was significantly associated with the TNM stage, tumor differentiation, and lymph node metastasis, indicating that the expression level of CD63 may negatively regulate the development and metastasis of TSCC.

Loss-of-function studies have benefited greatly from the use of RNA interference techniques. The utilization of
shRNAs, which enables stable gene silencing that is reversible and provides a method to examine the outcomes of temporary in vivo target inhibition, assess long-term phenotypes, and conduct pool-based forward genetic screening (24). The 293 cell line and its derivatives are commonly used as a vehicle in cell biology studies, owing to their high transfection efficiency. To identify the function of CD63 in the cell line TCA8113, three shRNA plasmids against CD63 gene were designed and constructed in the present study. Lipofectamine 2000 was used to transfect the shRNA plasmids into 293 cells, and the interference efficiency was evaluated using RT-qPCR and western blotting. The results confirmed that all three shRNA plasmids were able to greatly reduce the expression of CD63, with the plasmid CD63-RNAi-4041 was the most efficient in this regard. The interference values of CD63-RNAi-4041 at the mRNA and protein levels were 88 and $74 \%$, respectively $(\mathrm{P}<0.05)$. However, Stepanenko and Dmitrenko (25) revealed that 293 cells are tumorigenic, whereas acute changes to expression of the cancer-associated genes aggravate tumorigenicity 
A

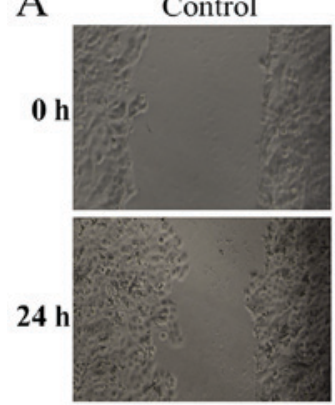

C

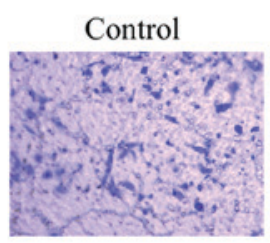

E

G

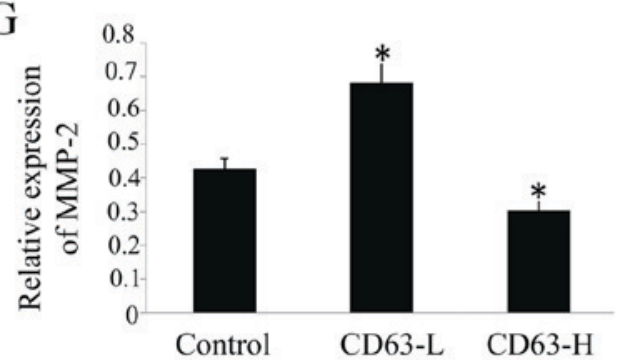

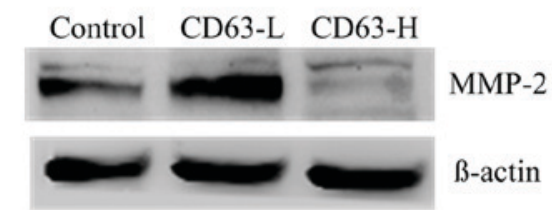

$\mathrm{F}$
CD63-H
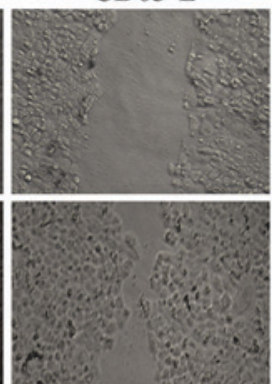

CD63-L
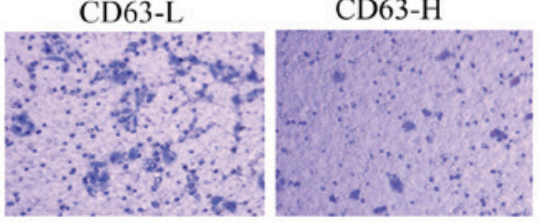

B
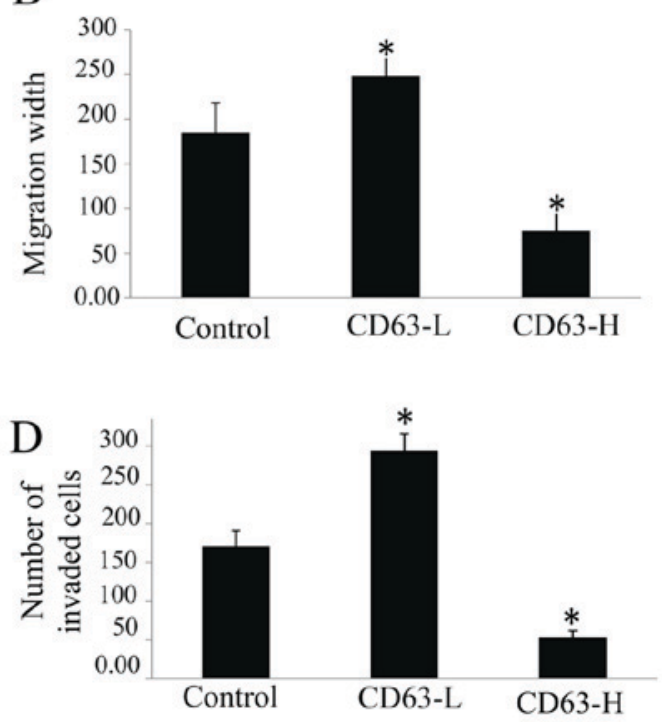

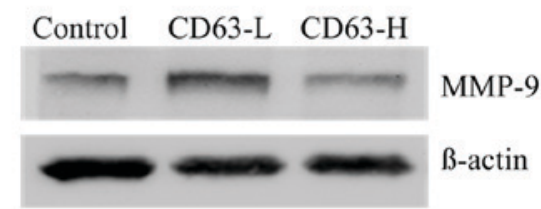

$\mathrm{H}$

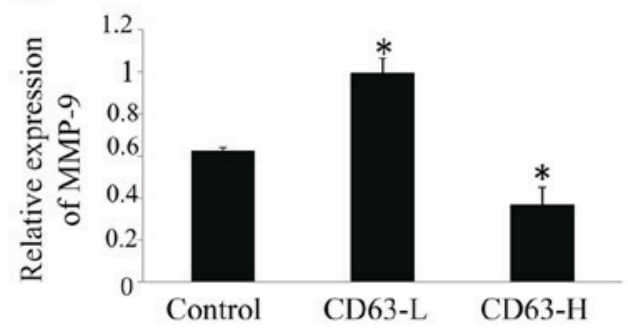

Figure 4. Role of CD63 in the migration and invasion of TCA8113 cells. (A) Images of the wound healing assay of TCA8113 cells (magnification, x100). (B) Quantitative analysis of the migration width of TCA8113 cells at $24 \mathrm{~h}$ in the wound-healing assay. ${ }^{*} \mathrm{P}<0.05$ vs. control. (C) The invasive ability of TCA8113 cells analyzed by Transwell assay (magnification, x100). (D) Number of invaded cells in 24 h. . P $<0.05$ vs. control. The expression of (E) MMP-2 and (G) MMP-9 with quantification of expression of (F) MMP-2 and (H) MMP-9 in CD63-silenced and CD63-overexpressing TCA8113 cells. * $<0.05$ vs. control. Control, TCA8113 cells untransfected exogenous CD63; CD63-L, low CD63 expression; CD63-H, high CD63 expression; MMP-9, matrix metalloproteinase-9.

by promoting chromosomal instability. Even the transfection of a stable empty vector can alter the karyotype and phenotype (25). In the present study, in order to exclude the cell karyotype and phenotype changes in 293 cells, the study further examined the interfering efficiency of the shRNA plasmids in TCA8113 cells. Therefore, any controversy from the usage of 293 cells could be dismissed.

A CD63-overexpressing plasmid (PEGFP-N3-CD63) was constructed using a PCR-based method. CD63-RNAi-4041 and PEGFP-N3-CD63 plasmids were transfected into TCA8113 cells, and G418 was used to screen stable cell lines. Stable TCA8113 cell lines were eventually obtained, and the CD63 expression level was detected by western blotting. Thus, CD63-silenced and CD63-overexpressing TCA8113 cells were generated, which were termed CD63-L and CD63-H, respectively.

Tumor growth and spread is a multistage process. First, normal cells undergo genetic changes that alter their phenotypes and enable their ability to spread and colonize, even to distant sites. A number of factors regulate tumor growth and spread, and interactions between the tumor and its microenvironment provide protein products that are crucial to each step of tumor progression (26). In addition, the expression of proteolytic enzymes is associated with metastatic phenotypes. For example, the MMPs, a family of degradative enzymes associated with malignancy, are involved in the degradation of the extracellular matrix, including the basement membrane, which is a specialized matrix composed of type IV collagen, laminin, entactin, proteoglycans and glycosaminoglycans (27). The ubiquitously present basement membrane serves as a barrier between tissue compartments, and if the integrity of the basement membrane is disrupted (which happens in invasive tumors), the disruption allows the tumor to spread locally and distantly $(28,29)$.

MMP-2 and MMP-9 serve notable roles in the degradation of the basement membrane. MMP-2 and MMP-9 are closely associated with tumor progression in human oral squamous cell carcinoma, and several studies indicate that these gelatinases are localized to the advancing tumor front, and have been implicated in metastatic dissemination (30-34). In the present study, 
western blot analysis of the expression of MMP-2 and MMP-9 in CD63-silenced and CD63-overexpressing TCA8113 cells revealed that when the expression of CD63 was silenced in CD63-L cells, the expressions of MMP-2 and MMP- 9 were increased by 60 and $61 \%$, respectively, whereas the expression of these proteins were reduced by 29 and $41 \%$, respectively, in CD63-H cells. Considering that the changes in expression of MMP-2 and MMP-9 may alter the biological behavior of TCA8113 cells, wound-healing and Transwell invasion assays were used to measure the migratory and invasive ability of TCA8113 cells.

In the wound-healing assay, the wound-healing ability of the control cells was $73 \%$ lower than that of the CD63-L cells, but $59 \%$ higher than that of the CD63-H cells. In the Transwell invasion assay, TCA8113 cells degraded the Matrigel matrix and passed through the $8.0-\mu \mathrm{m}$-pore membrane through cellular plasticity. The knockdown of CD63 enhanced the invasive ability of TCA8113 cells.

In conclusion, the findings of the present study indicate that CD63 may serve an inhibitory role in the malignancy and lymph node metastasis of TSCC, and may have potential applications in the prediction of prognosis and gene therapy for TSCC patients.

\section{Acknowledgements}

The authors would like to thank Professor Rongjian Su and Professor Cuifen Bao of Jinzhou Medical University for their technical guidance. The authors acknowledge the Pathology Department of the First Affiliated Hospital of Jinzhou Medical University for providing human tongue and TSCC tissue specimens.

\section{Funding}

The present study was supported by grants from the National Nature Science Foundation of China (no. 81201285); the National Nature Science Foundation of Liaoning Province (no. 20170540398); the Excellent Talents Project in Colleges and Universities of the Liaoning Province Foundation (no. LJQ2015067), and the Quanmin Oral Graduate Sci-tech Innovation Foundation, the President Fund of Jinzhou Medical University (project no. QM2014003).

\section{Availability of data and materials}

The datasets used and/or analyzed during the current study are available from the corresponding author on reasonable request.

\section{Authors' contributions}

WZ and XL conceived and designed the experiments. WHL performed the experiments and wrote the paper. XLZ and MLH analyzed the data.

\section{Ethics approval and consent to participate}

The present study was approved by the Ethics Committees of Jinzhou Medical University (approval nos. 20140005 and 20171105). Written informed consent was obtained from all patients.

\section{Consent for publication}

Written informed consent for the publication of data was obtained from all patients.

\section{Competing interests}

The authors declare that they have no competing interests.

\section{References}

1. Jemal A, Bray F, Center MM, Ferlay J, Ward E and Forman D: Global cancer statistics. CA Cancer J Clin 61: 69-90, 2011.

2. Siegel RL, Miller KD and Jemal A: Cancer statistics, 2015. CA Cancer J Clin 65: 5-29, 2015.

3. Sano D and Myers JN: Metastasis of squamous cell carcinoma of the oral tongue. Cancer Metastasis Rev 26: 645-662, 2007.

4. Neville BW and Day TA: Oral cancer and precancerous lesions. CA Cancer J Clin 52: 195-215, 2002.

5. Ren W, Lian P, Cheng L, Du P, Guan X, Wang H, Ding L, Gao Z, Huang X, Xiao F, et al: FHL1 inhibits the growth of tongue squamous cell carcinoma cells via G1/S cell cycle arrest. Mol Med Rep 12: 3958-3964, 2015.

6. Liu Z, He Q, Ding X, Zhao T, Zhao L and Wang A: SOD2 is a $\mathrm{C}$-myc target gene that promotes the migration and invasion of tongue squamous cell carcinoma involving cancer stem-like cells. Int J Biochem Cell Biol 60: 139-146, 2015.

7. Jia LF, Gan YH and Yu GY: Relationships between microRNA expressions and prognosis in patients with tongue squamous cell carcinoma and the mechanisms microRNA regulating tongue squamous cell carcinoma biological behavior. Beijing Da Xue Xue Bao Yi Xue Ban 48: 5-9, 2016 (In Chinese).

8. Hotta H, Ross AH, Huebner K, Isobe M, Wendeborn S, Chao MV, Ricciardi RP, Tsujimoto Y, Croce CM and Koprowski H: Molecular cloning and characterization of an antigen associated with early stages of melanoma tumor progression. Cancer Res 48: 2955-2962, 1988.

9. Maecker HT, Todd SC and Levy S: The tetraspanin superfamily: Molecular facilitators. FASEB J 11: 428-442, 1997.

10. Pols MS and Klumperman J: Trafficking and function of the tetraspaninCD63. Exp Cell Res 315: 1584-1592, 2009.

11. Kobayashi T, Vischer UM, Rosnoblet C, Lebrand C, Lindsay M, Parton RG, Kruithof EK and Gruenberg J: The tetraspanin CD63/lamp3 cycles between endocytic and secretory compartments in human endothelial cells. Mol Biol Cell 11: 1829-1843, 2000.

12. Kang M, Ryu J, Lee D, Lee MS, Kim HJ, Nam SH, Song HE, Choi J, Lee GH, Kim TY, et al: Correlations between transmembrane 4 L6 family member 5 (TM4SF5), CD151, and CD63 in liver fibrotic phenotypes and hepatic migration and invasive capacities. PLoS One 9: e102817, 2014.

13. Hunziker W and Geuze HJ: Intracellular trafficking of lysosomal membrane proteins. Bioessays 18: 379-389, 1996.

14. Atkinson B, Ernst CS, Ghrist BF, Herlyn M, Blaszczyk M, Ross AH, Herlyn D, Steplewski Z and Koprowski H: Identification of melanoma-associated antigens using fixed tissue screening of antibodies. Cancer Res 44: 2577-2581, 1984.

15. Jang HI and Lee H: A decrease in the expression of CD63 tetraspanin protein elevates invasive potential of human melanoma cells. Exp Mol Med 35: 317-323, 2003.

16. Radford KJ, Thorne RF and Hersey P: Regulation of tumor cell motility and migration by CD63 in a human melanoma cell line. J Immunol 158: 3353-3358, 1997.

17. Kwon MS, Shin SH, Yim SH, Lee KY, Kang HM, Kim TM and Chung YJ: CD63 as a biomarker for predicting the clinical outcomes in adenocarcinoma of lung. Lung Cancer 57: 46-53, 2007.

18. Sauer G, Kurzeder C, Grundmann R, Kreienberg R, Zeillinger R and Deissler $\mathrm{H}$ : Expression of tetraspanin adaptor proteins below defined threshold values is associated with in vitro invasiveness of mammary carcinoma cells. Oncol Rep 10: 405-410, 2003.

19. Sordat I, Decraene C, Silvestre T, Petermann O, Auffray C, Piétu G and Sordat B: Complementary DNA arrays identify CD63 tetraspanin and alpha3 integrin chain as differentially expressed in low and high metastatic human colon carcinoma cells. Lab Invest 82: 1715-1724, 2002. 
20. Shear M: The aggressive nature of the odontogenic keratocyst: Is it a benign cystic neoplasm? Part 3. Immunocytochemistry of cytokeratin and other epithelial cell markers. Oral Oncol 38: 407-415, 2002

21. Livak KJ and Schmittgen TD: Analysis of relative gene expression data using real-time quantitative PCR and the 2(-Delta Delta C(T)) method. Methods 25: 402-408, 2001.

22. Woegerbauer M, Thurnher D, Houben R, Pammer J, Kloimstein P, Heiduschka G, Petzelbauer P and Erovic BM: Expression of the tetraspanins CD9, CD37, CD63, and CD151 in Merkel cell carcinoma: Strong evidence for a posttranscriptional fine-tuning of CD9 gene expression. Mod Pathol 23: 751-762, 2010.

23. Chen Z, Gu S, Trojanowicz B, Liu N, Zhu G, Dralle H and Hoang-Vu C: Down-regulation of TM4SF is associated with the metastatic potential of gastric carcinoma TM4SF members in gastric carcinoma. World J Surg Oncol 9: 43, 2011.

24. Fellmann C and Lowe SW: Stable RNA interference rules for silencing. Nat Cell Biol 16: 10-18, 2014.

25. Stepanenko AA and Dmitrenko VV: HEK293 in cell biology and cancer research: Phenotype, karyotype, tumorigenicity, and stress-induced genome-phenotype evolution. Gene 569: 182-190, 2015.

26. Nelson AR, Fingleton B, Rothenberg ML and Matrisian LM: Matrix metalloproteinases: Biologic activity and clinical implications. J Clin Oncol 18: 1135-1149, 2000.

27. Yurchenco PD and Schittny JC: Molecular architecture of basement membranes. FASEB J 4: 1577-1590, 1990.

28. Barsky SH, Siegal GP, Jannotta F and Liotta LA: Loss of basement membrane components by invasive tumors but not by their benign counterparts. Lab Invest 49: 140-147, 1983.
29. Liotta LA, Tryggvason K, Garbisa S, Hart I, Foltz CM and Shafie S: Metastatic potential correlates with enzymatic degradation of basement membrane collagen. Nature 284: 67-68, 1980.

30. Kusukawa J, Sasaguri Y, Shima I, Kameyama T and Morimatsu M: Expression of matrix metalloproteinase-2 related to lymph node metastasis of oral squamous cell carcinoma. A clinicopathologic study. Am J Clin Pathol 99: 18-23, 1993.

31. Kawamata H, Uchida D, Hamano H, Kimura-Yanagawa T, Nakashiro KI, Hino S, Omotehara F, Yoshida $\mathrm{H}$ and Sato M: Active-MMP2 in cancer cell nests of oral cancer patients: Correlation with lymph node metastasis. Int J Oncol 13: 699-704, 1998.

32. Ikebe T, Shinohara M, Takeuchi H, Beppu M, Kurahara S, Nakamura $S$ and Shirasuna K: Gelatinolytic activity of matrix metalloproteinase in tumor tissues correlates with the invasiveness of oral cancer. Clin Exp Metastasis 17: 315-323, 1999.

33. Hong SD, Hong SP, Lee JI, and Lim CY: Expression of matrix metalloproteinase-2 and -9 in oral squamous cell carcinomas with regard to the metastatic potential. Oral Oncol 36: 207-213, 2000.

34. Yorioka CW, Coletta RD, Alves F, Nishimoto IN, Kowalski LP and Graner E: Matrix metalloproteinase-2 and -9 activities correlate with the disease-free survival of oral squamous cell carcinoma patients. Int J Oncol 20: 189-194, 2002.

This work is licensed under a Creative Commons Attribution-NonCommercial-NoDerivatives 4.0 International (CC BY-NC-ND 4.0) License. 